\title{
OPEN KIAA0319 influences cilia length, cell migration and mechanical cell-substrate interaction
}

\author{
Rebeca Diaz ${ }^{1,4}$, Nils M. Kronenberg ${ }^{2,3,4}$, Angela Martinelli ${ }^{1}$, Philipp Liehm², \\ Andrew C. Riches ${ }^{1}$, Malte C. Gather ${ }^{2,3}$ \& Silvia Paracchini ${ }^{1 \bowtie}$
}

Following its association with dyslexia in multiple genetic studies, the KIAA0319 gene has been extensively investigated in different animal models but its function in neurodevelopment remains poorly understood. We developed the first human cellular knockout model for KIAA0319 in RPE1 retinal pigment epithelia cells via CRISPR-Cas9n to investigate its role in processes suggested but not confirmed in previous studies, including cilia formation and cell migration. We observed in the KIAA0319 knockout increased cilia length and accelerated cell migration. Using Elastic Resonator Interference Stress Microscopy (ERISM), we detected an increase in cellular force for the knockout cells that was restored by a rescue experiment. Combining ERISM and immunostaining we show that RPE1 cells exert highly dynamic, piconewton vertical pushing forces through actin-rich protrusions that are surrounded by vinculin-rich pulling sites. This protein arrangement and force pattern has previously been associated to podosomes in other cells. KIAA0319 depletion reduces the fraction of cells forming these actin-rich protrusions. Our results suggest an involvement of KIAA0319 in cilia biology and cell-substrate force regulation.

Dyslexia is a neurodevelopmental disorder that affects around 5\% of school-aged children and refers to unexpected difficulties in learning to read ${ }^{1}$. Dyslexia is high heritable (up to $70 \%$ ). Genetic studies, mainly in familybased samples, have focused their attention on genes that play a role in neurodevelopment, including DYX1C1, $D C D C 2, R O B O 1$ and KIAA03192. Functional analysis of these genes have largely contributed to shape hypotheses aimed at explaining the neurobiology of dyslexia. Initial in utero gene silencing experiments in rats for these genes provided strong support for the neuronal migration hypothesis ${ }^{3}$ first proposed in the eighties ${ }^{4}$. This hypothesis is based on the observation of subtle cortical anomalies, i.e. heterotopias and microgyrias, in postmortem brains from individuals with dyslexia $(n=8)$. Such anomalies are likely to be the result of neuronal migration defects. However, knockout mouse models for DYX1C1, DCDC2 and KIAA0319 did not exhibit cortical alterations ${ }^{5}$, although heterotopias were observed in rats subjected to in utero knockdown of Dyx $1 \mathrm{cl}^{6}$. The discordance between knockdown experiments in rat and knockout mouse models has been explained by species-specific effects, compensatory mechanisms in mice, or artefacts in shRNA experiments, and has been highlighted by recent reviews of the literature, providing interpretations either in support or raising doubts about the neuronal migration hypothesis ${ }^{5,7,8}$.

In parallel, emerging evidence supports roles of DCDC2, DYX1C1, ROBO1 and KIAA0319 in cilia biology ${ }^{2}$. Transcriptomic studies showed differential expression for these genes in ciliated tissue ${ }^{9-11}$. Beyond these studies, a role of KIAA0319 in cilia biology has not been described yet, but cellular and animal knockouts for DCDC2 and DYX1C1 presented cilia defects. Mutations in DYX1C1 and DCDC2 have been identified in patients with ciliopathies, a group of disorders caused by defective cilia and often characterised by alterations in body asymmetry. ROBO1 has been shown to localize to the cilium of mouse embryonic interneurons. Cilia biology has been proposed as a molecular link to explain the atypical brain asymmetries which are consistently reported for neurodevelopmental disorders, such as dyslexia ${ }^{12,13}$.

KIAA0319 encodes a transmembrane protein with five PKD domains ${ }^{14,15}$ (Fig. 1A). Such structures have been previously found in cell surface proteins and are known to be involved in cell-cell and cell-matrix interactions ${ }^{16}$ but the cellular function of KIAA0319 remains largely uncharacterised ${ }^{5}$. KIAA0319 has also been suggested to play a role in signalling pathways ${ }^{17}$ and in axon growth inhibition ${ }^{18}$. A gene expression analysis in zebrafish

\footnotetext{
${ }^{1}$ School of Medicine, University of St Andrews, St Andrews KY16 9TF, UK. ${ }^{2}$ SUPA, School of Physics and Astronomy, University of St Andrews, St Andrews KY16 9SS, UK. ${ }^{3}$ Department of Chemistry, Humboldt Centre for Nano- and Biophotonics, University of Cologne, 50939 Cologne, Germany. ${ }^{4}$ These authors contributed equally: Rebeca Diaz and Nils M. Kronenberg. ${ }^{\bowtie}$ email: mcg6@st-andrews.ac.uk; sp58@st-andrews.ac.uk
} 
A

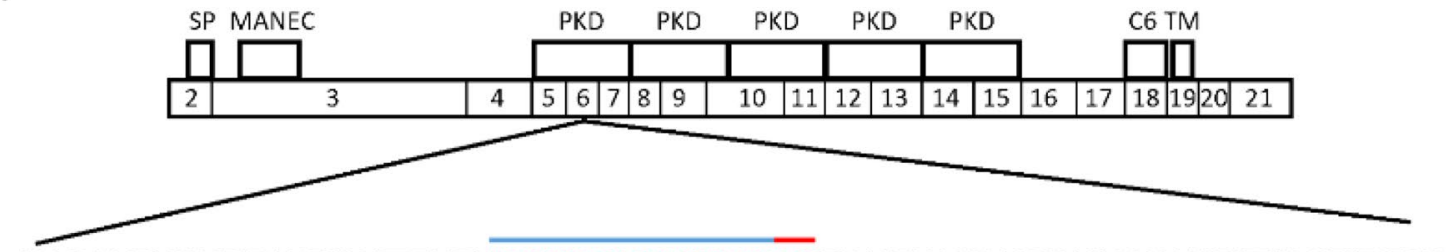

AAACAACCTACAACTATGAATGGAATTAATAAGCCACCCCACAGACTACCAAGGTGAAATAAAACAAGGACACAAGCAAACTCTTAACCTCTCTCAA TTTGTTGGATGTTGATACTTACCTTAAATTATTCGGTGGGGTGTCTGATGGTTCCACTTTATTTTGTTCCTGTGTTCGTTTGAGAATTGGAGAGAGTT

\section{TTYNYEWNLISHPTDYQGEIKQGHKQTLNLSQ}

B

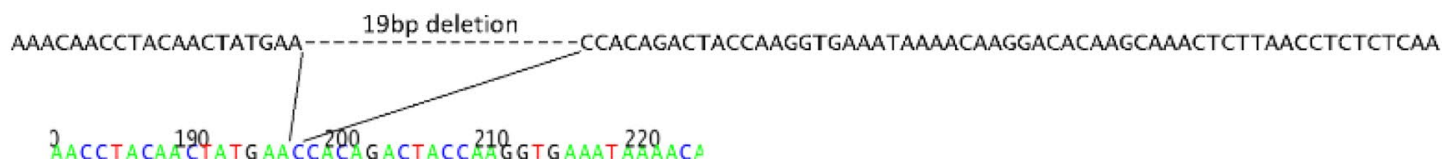

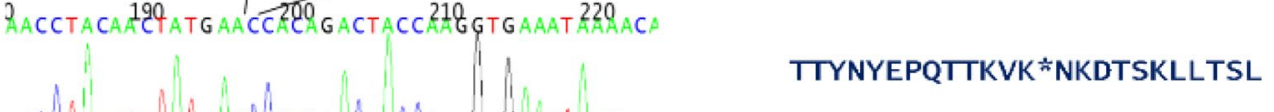
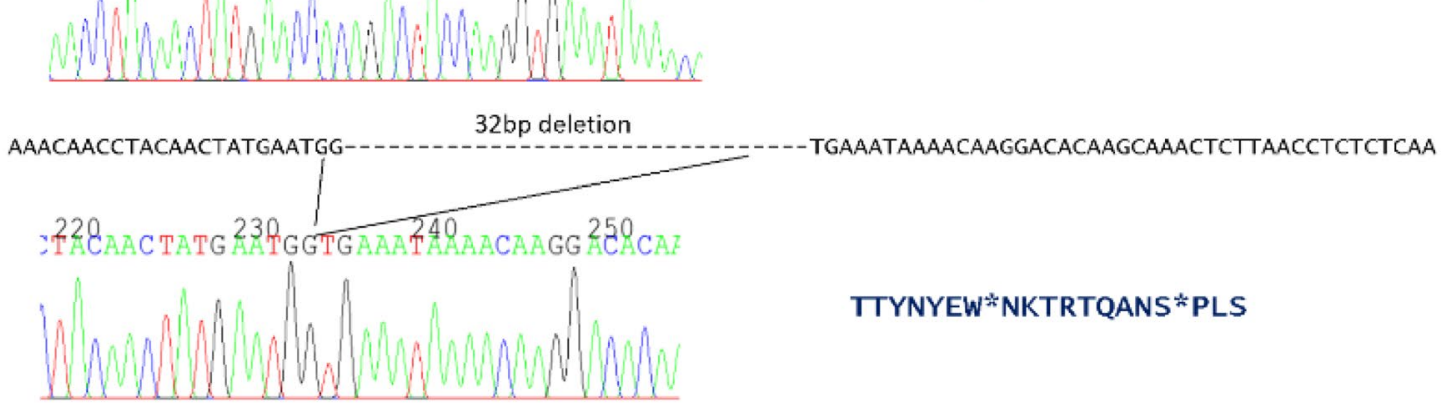

TTYNYEW*NKTRTQANS*PLS

C
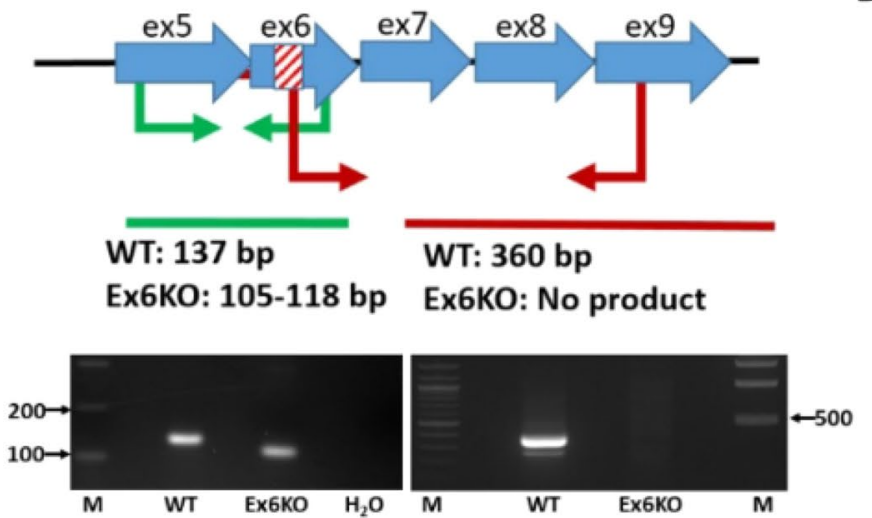

D

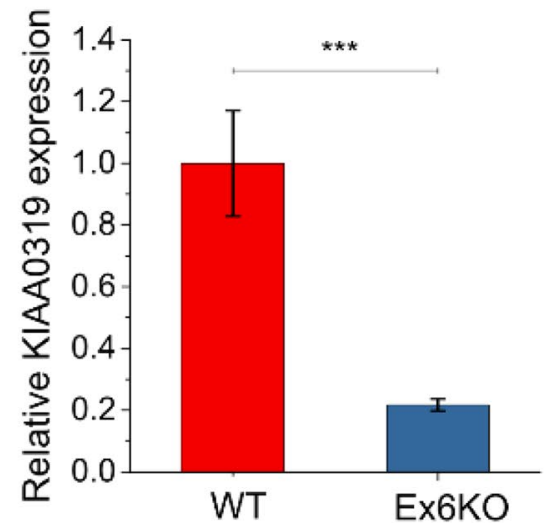

Figure 1. Generation of a cellular KIAA0319 knockout. (A) Top: Structure of Human KIAA0319 (based on ${ }^{15}$ and Ensembl release $94^{21}$ ). The diagram shows the correspondence between protein domains and coding exons in KIAA0319. Signal peptide (SP), MANEC domain (MANEC), PKD domains (PKD), cysteine residues (C6) and transmembrane domain (TM) are indicated. Bottom: full DNA sequence of KIAA0319 exon 6 with target sequences for the gRNAs indicated with blue lines. Red lines show the position of the PAM sequences. Translated sequence of amino acids for the targeted exon is shown below the DNA sequence. (B) Chromatograms of the deletions found in Ex6KO and translated corresponding amino acids for wild type and knockout cell line. Asterisks indicate premature stop codons. (C) Results of the PCR screening to confirm the deletions in Ex6KO. The cartoon on the left represents the screening strategy. Two sets of primers were designed to give different bands in the WT and KO. The stripped area indicates the 19 and 32 base pair (bp) deletions in the exon 6 of KIAA0319. The first set of primers (Ex_6R and Ex_5F) amplifies the region around the deletion and therefore a smaller band is expected for the KO (105-118 bp) compared to the WT (137 bp). The second pair (Ex9R/Ex6delF) has one primer mapping within the deletion. PCR is expected to give a band of $360 \mathrm{bp}$ in the WT and no product in the KO. Images below confirm the expected results for both pairs. Full-length images are presented in Supplementary Fig. S3. (D) Quantification of KIAA0319 mRNA in WT and Ex6KO by qPCR. KIAA0319 expression is significantly lower in Ex6KO (Student's $t$-test: $p \leq 0.0001$ ), consistent with nonsense mediated decay of the mRNA caused by the deletion. Origin 2018 version 95E (https://www.originlab.com/ 20182 was used for data plotting and significance testing. 
showed very high expression in the first hours of development and specific signal in defined embryonic structures, including the notochord and the developing eye and otic vesicles ${ }^{19}$. Moreover, in support of the importance of KIAA0319 in brain function, a recent study suggests a possible role of KIAA0319 in Alzheimer's disease ${ }^{20}$.

In spite of these intensive efforts, the precise role of KIAA0319 remains unexplained. The initial neuronal migration hypothesis is not consistently supported, and direct evidence for a role in cilia biology, similarly to other genes implicated in dyslexia, has not been described yet. To address this issue, we generated the first cellular knockout model of KIAA0319 in human cells and used this model to specifically investigate the role of KIAA0319 in cilia biology and cell migration, addressing the two main hypotheses currently proposed. We used retinal pigmented epithelial cells (RPE1), which are particularly suitable to study cilia, and studied their mechanobiology using a range of assays including the recently introduced Elastic Resonator Interference Stress Microscopy (ERISM) $)^{22,23}$ that allows for continuous imaging of cell forces with high spatial resolution and over extended periods of time.

Our KIAA0319 knockout model presents longer cilia, changed migratory behaviour and increased cellular force exertion. In addition, we observe actin-rich cell protrusions surrounded by vinculin-rich pulling sites-a protein arrangement and force pattern that has previously been associated with podosomes, a cellular adhesion structure that performs mechanosensing via the exertion of oscillating, vertical forces ${ }^{24}$. Our data further indicate that KIAA0319 knockout cells present fewer of these protrusions than control cells.

\section{Results}

Generation of KIAA0319 KO in RPE1 cell lines. We generated KIAA0319 knockout RPE1 cells with CRISPR-Cas9n based genome editing. The KIAA0319 main isoform (NM_014809) consists of 21 exons and spans $102 \mathrm{~kb}$ of human chromosome 6 (Fig. 1A). We generated a biallelic knockout (Ex6KO) by causing deletions that introduce premature stop codons at exon 6 of KIAA0319 using paired gRNAs (Fig. 1B). The deletion was confirmed by RT-PCR (Fig. 1C). Transcript quantification by qRT-PCR shows that KIAA0319 expression in Ex6KO is five-times lower than the wild-type $(t$-test, $p \leq 0.001)$ which is consistent with degradation of the transcript by nonsense-mediated decay ${ }^{25}$ (Fig. 1D). We characterised the KIAA0319 knockout to address two specific hypotheses emerging from the literature. Specifically, we tested whether KIAA0319 might play a role in (i) cilia and (ii) cell migration.

KIAA0319 knockout cells form longer primary cilia. We measured cilia length in RPE1 wild type (WT) and Ex6KO cells by staining of the cilium-specific protein ARL13B and analysis of epi-fluorescence images (Fig. 2A). While a similar fraction of WT and Ex6KO cells formed cilia (WT: 379/571, 68\%; Ex6KO: 271/383, $70 \%$ ), the cilia in Ex6KO were significantly longer than in the wild type (mean \pm SEM: WT: $4.5 \mu \mathrm{m} \pm 0.1 \mu \mathrm{m}$, $n=129$; Ex6KO: $6.1 \mu \mathrm{m} \pm 0.2 \mu \mathrm{m}, n=104$; $t$-test: $p \leq 0.001$; Fig. $2 \mathrm{~B}$ ).

Migration speed, cell morphology, and force exertion are altered in KIAA0319 knockout cells. The second hypothesis we investigated was the role of KIAA0319 in cell migration. We started by comparing WT and KO with a scratch assay on confluent layers of cells to test collective cell migration. The assays did not reveal a significant difference in the capacity to cover the empty space between WT and Ex6KO cells after $24 \mathrm{~h}$ (mean cell coverage \pm SEM: WT: $27.4 \% \pm 4.2 \%, n=3$; Ex6KO: $30.2 \% \pm 3.5 \%, n=3$; $t$-test: $p=0.63$; Fig. S1).

Next, we turned to investigations on the single cell level, characterizing migration speed and cell morphology through detailed analysis of phase contrast microscopy and, in parallel, mapping the mechanical forces exerted by the cells using ERISM. For ERISM, cells are cultured on substrates that consist of a layer of an ultra-soft elastomer situated between two semi-transparent, mechanically flexible gold mirrors, which form an optical micro-cavity. Mechanical force and stress exerted by cells cause local deformations of the soft micro-cavity (the effective stiffness of cavities used in this study is $6 \mathrm{kPa}$ ). This in turn leads to local shifts in cavity resonance that are analysed by optical modelling to compute a high-resolution displacement map with $\mu \mathrm{m}$ lateral resolution and $n$ m vertical resolution ${ }^{23}$, which allows for the detection of forces in the piconewton range. In our earlier work $^{22}$, ERISM has been extensively calibrated (through application of well-defined forces by an atomic force microscope) and validated (through measurements on widely studied cell lines). The substrates used for ERISM are semi-transparent, thus allowing to combine force mapping with high-resolution imaging.

WT and Ex6KO cells were plated on ERISM substrates at a density low enough to ensure non-confluency and thus allow the behaviour of individual cells to be analysed (Fig. 3A). Quantitative image analysis showed that Ex6KO cells covered a smaller surface area than WT cells (mean cell area \pm SEM: WT: $2052 \mu \mathrm{m}^{2} \pm 91 \mu \mathrm{m}^{2}$, $n=36$; Ex6KO: $1295 \mu \mathrm{m}^{2} \pm 65 \mu \mathrm{m}^{2}, n=36$; $t$-test: $p \leq 0.001$; Fig. $3 \mathrm{~B}$ ), even though the shape and morphology of the cells did not differ. The displacement maps recorded with ERISM (Fig. 3A) revealed that cells from both lines generated similar spatial patterns of force on their substrate (even though absolute forces were markedly different, see next section): pulling was focused around the two long ends of the cells, perpendicular to the direction of migration (cells were polarised in a way that the nucleus was positioned posterior to the direction of migration). Downward compression was observed underneath the centre of the cells. This displacement pattern is a fingerprint for the exertion of contractile forces by adherent cells ${ }^{22}$.

The migratory behaviour and the associated dynamics of force exertion of WT and Ex6KO cells were then investigated by taking time-lapse measurements of phase contrast and ERISM displacement maps in 5-min intervals over a time span of $17 \mathrm{~h}$ (Movies S1, S2). The average speed of single cell migration was significantly higher for Ex6KO than for WT cells (mean speed \pm SEM: WT: $0.32 \mu \mathrm{m} \mathrm{min}^{-1} \pm 0.02 \mu \mathrm{m} \mathrm{min}^{-1}, n=29$; Ex6KO: $0.40 \mu \mathrm{m} \mathrm{min}{ }^{-1} \pm 0.02 \mu \mathrm{m} \mathrm{min}^{-1}, n=24, t$-test: $p=0.02$; only cells with free movement for $\geq 2 \mathrm{~h}$ were included in analysis; Fig. 3C). To assess the force exerted by cells, we computed the total volume by which each cell indents into the substrate and used this as a proxy for the applied force. (For further explanation of this approach, please 
A
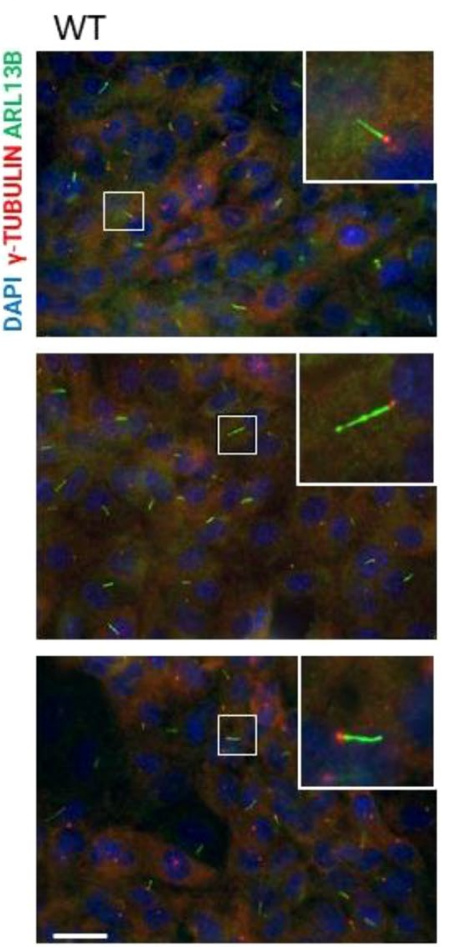

Ex6KO
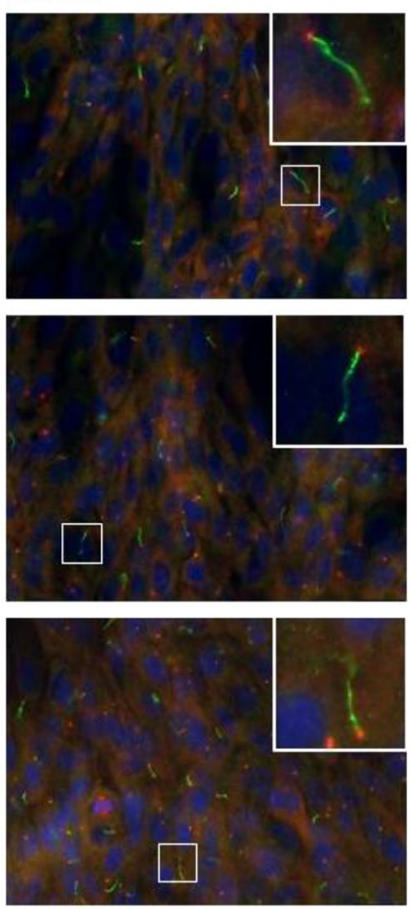

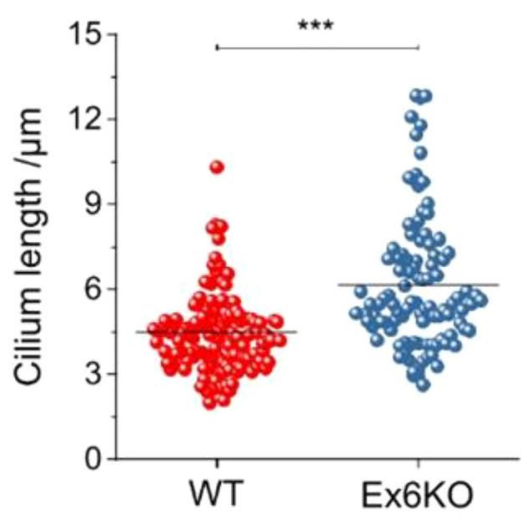

Figure 2. Analysis of the cilia length. (A) Representative immunofluorescence images of RPE1 wild type and Ex6KO, stained for cilia marker Arl13b (green), centrosomal marker $\gamma$-tubulin (red), and DAPI (blue). Insets show magnifications of the cilia indicated with small white squares. (B) Plot of the cilia length for wild type $(n=129)$ and Ex6KO cells $(n=104)$. Groups were compared using the Student's $t$-test $\left({ }^{* *} p \leq 0.001\right)$. Origin 2018 version 95E (https://www.originlab.com/2018) was used for data plotting and significance testing. Scale bar, $10 \mu \mathrm{m}$.

see our earlier publications on ERISM $^{22,23}$ ). Comparing the temporal averages of applied force during migration showed that Ex6KO cells exerted significantly stronger contractile forces on the substrate than WT cells (mean indented volume \pm SEM: WT: $168 \mu \mathrm{m}^{3} \pm 16 \mu \mathrm{m}^{3}, n=29$; Ex6KO: $273 \mu \mathrm{m}^{3} \pm 24 \mu \mathrm{m}^{3}, n=24$; $t$-test: $p=0.0006$; only cells with free movement for $\geq 2 \mathrm{~h}$ were included in analysis; Fig. 3D).

Next, we investigated changes in migration speed and exerted force over time (Fig. S2A). WT and Ex6KO cells showed no differences in how migration speed and applied force fluctuated when normalising data to the respective means (Fig. S2B,C). For both WT and Ex6KO cells, intervals of increased migration speed correlated with drops in cell forces (anti-correlation between the first time derivative of speed and the first time derivative of mechanical activity). Again, there was no significant difference in this correlation between the two groups (Fig. S2D-G). Furthermore, the straightness of the migration was not affected by the KIAA0319 knockout (Fig. S2H).

To validate our findings of the impact of KIAA0319 on cell area and cell force, we conducted a rescue experiment by generating an Ex6KO cell line with stable expression of KIAA0319-GFP fusion protein (Ex6KO K-GFP; Fig. 4A). We also generated a control line of RPE1 WT cells with the same construct (WT K-GFP). Even though the KIAA0319 rescue did not recover the reduction in cell area seen for Ex6KO cells [mean cell area \pm SEM: WT: $2315 \mu \mathrm{m}^{2} \pm 200 \mu \mathrm{m}^{2}, n=16$; WT K-GFP: $2299 \mu \mathrm{m}^{2} \pm 107 \mu \mathrm{m}^{2}, n=20$; Ex6KO: $1565 \mu \mathrm{m}^{2} \pm 123 \mu \mathrm{m}^{2}, n=23$; Ex6KO K-GFP: $1297 \mu \mathrm{m}^{2} \pm 131 \mu \mathrm{m}^{2}, n=17$; Fig. $4 \mathrm{~B}$ ], the level of cell force was restored in Ex6KO K-GFP cells [mean indented volume \pm SEM: WT: $115 \mu \mathrm{m}^{3} \pm 14 \mu \mathrm{m}^{3}, n=16$; WT K-GFP: $96 \pm 9 \mu \mathrm{m}^{3}, n=19$; Ex6KO: $186 \pm 20 \mu \mathrm{m}^{3}, n=24$; Ex6KO K-GFP: $125 \pm 16 \mu \mathrm{m}^{3}, n=16$; $t$-test (WT vs. Ex6KO): $p=0.01, t$-test(WT vs. Ex6KO K-GFP): $p=0.67$; Fig. $4 \mathrm{C})$ ]. In both the transfected WT and Ex6KO cells, KIAA0319-GFP is mainly concentrated in small dots around the nucleus and on the cell edge, suggesting localisation to the secretion pathway and to the membrane, respectively (Fig. S4). Previous studies have shown that beside the plasma membrane, KIAAA0319 localises to the ER and to the trans-Golgi network when overexpressed ${ }^{15,26}$.

RPE1 KIAA0319 WT and Ex6KO show different fine patterns of force exertion. Given the differences in cilia length, cell area, migration speed and exerted force, we reasoned that KIAA0319 knockout might affect cytoskeleton dynamics. To test this hypothesis, we took phase contrast and ERISM time-lapse measurements of migrating WT and Ex6KO cells at 5 s intervals (Movies S3, S4), and fixed and immunostained the cells for actin and vinculin immediately after the time-lapse.

For further analysis, spatial Fourier-filtering of ERISM maps was used to filter out broad deformation features associated with the overall contractility of cells and thus resolve finer details linked to interaction of sub-cellular 
A

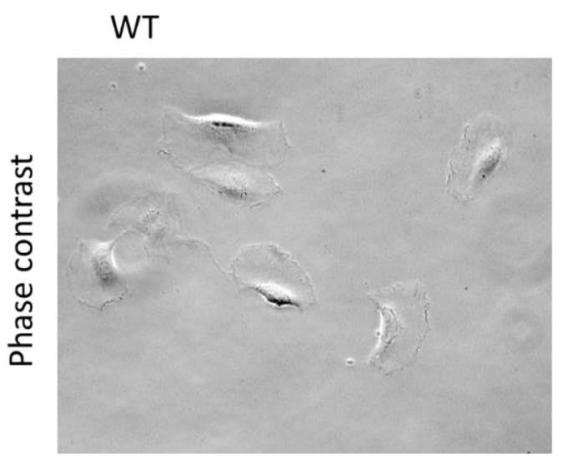

\section{Ex6KO}
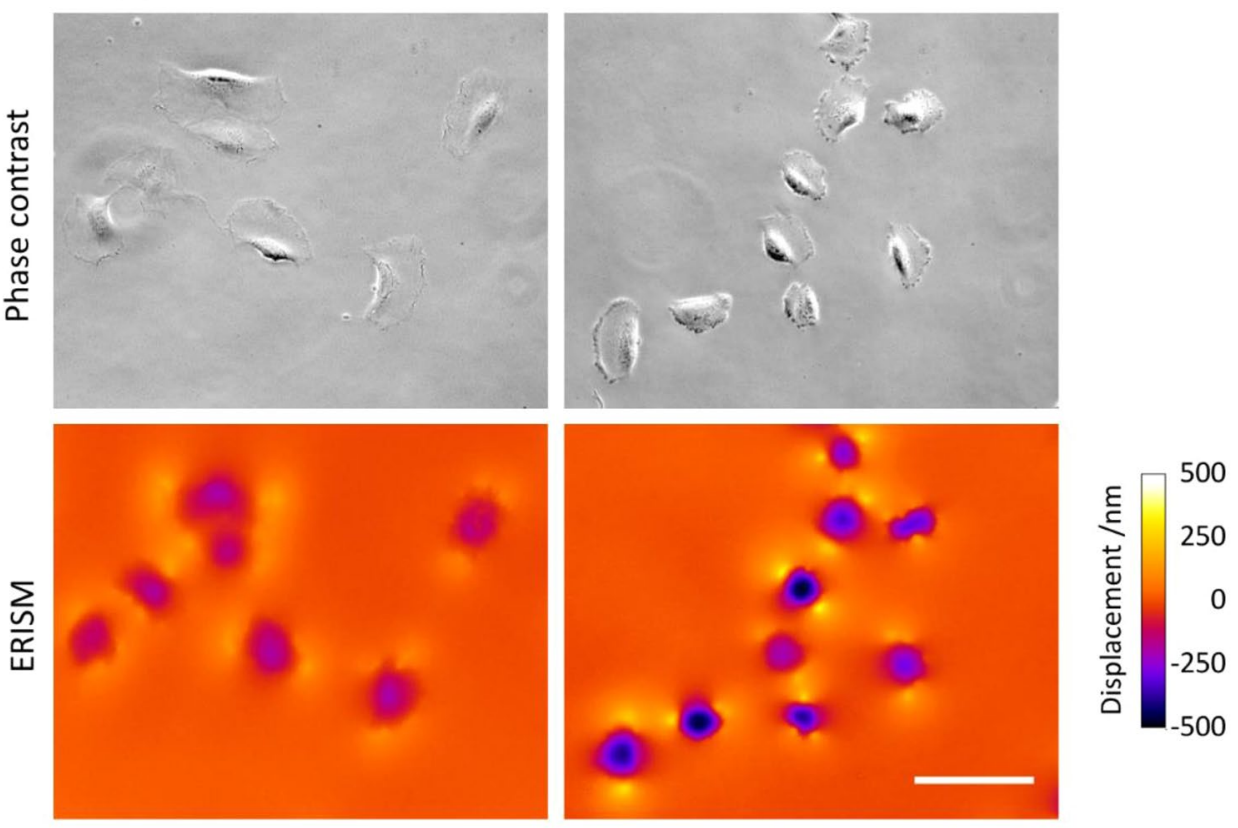

B

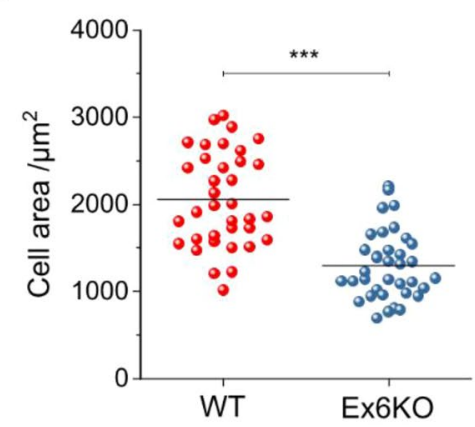

C

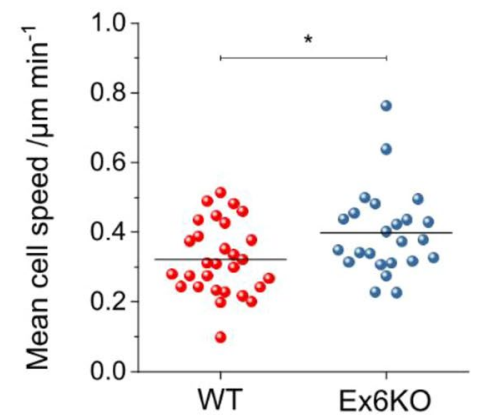

D

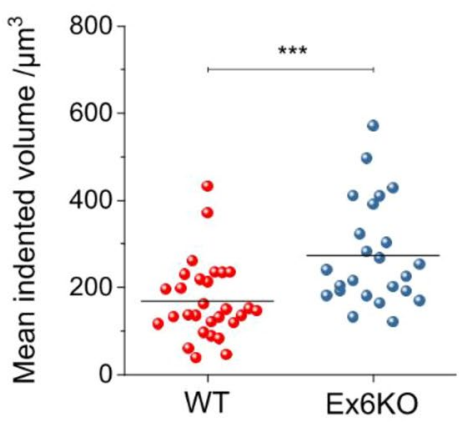

Figure 3. Analysis of mechanical activity of RPE1 WT and Ex6KO cells during migration on an ERISM micro-cavity. (A) Phase contrast (upper row) and ERISM micro-cavity displacement maps (lower row) of WT (left) and Ex6KO (right) cells. (B) Comparison of the surface area covered by WT $(n=36)$ and Ex6KO $(n=36)$ cells types. (C) Comparison of mean speed of WT $(n=29)$ and Ex6KO $(n=24)$ cells. (D) Comparison of mean indented volume of WT $(n=29)$ and Ex6KO $(n=24)$ cells. Only cells with free movement for $\geq 2 \mathrm{~h}$ were included in analysis of speed and indented volume. Plots in (B,D,E) show all measured data points and the mean (line). Groups were compared using the Student's $t$-test $\left({ }^{*} p \leq 0.05,{ }^{* *} p \leq 0.01,{ }^{* *} p \leq 0.001\right)$. Origin 2018 version $95 \mathrm{E}$ (https://www.originlab.com/2018) was used for data plotting and significance testing. Scale bar, $50 \mu \mathrm{m}$.

components, e.g. focal adhesions or podosomes, with the substrate ${ }^{22}$. (For further discussion on the displacement fine-structure in Fourier-filtered displacement maps see Fig. S5). Figure 5A shows phase contrast images, Fourier-filtered ERISM maps and immunofluorescence microscopy images for a WT and Ex6KO cell. The Fourier-filtered displacement maps of both cells showed numerous small push-pull features that co-localised with vinculin-rich areas in the immunofluorescence microscopy images (see insets ii and iii to Fig. 5A for examples of this feature). Vinculin is enriched in the centre between pulling (red areas in Fourier-filtered ERISM maps) and pushing (green areas). The actin fibres are connected to vinculin. Push-pull features in Fourier-filtered ERISM maps were previously attributed to focal adhesions transmitting horizontal forces that are generated by the actin cytoskeleton to the substrate ${ }^{22}$. In agreement with these earlier observations, the axes defined by the push-pull features co-aligned with the actin fibres that connect different vinculin-rich sites (best visible in the Ex6KO cell in Fig. 5A, Supplementary Fig. S5). This push-pull behaviour is also consistent with earlier observations of torque being applied by focal adhesions ${ }^{27}$.

The formation and alignment of stress fibres was less distinct in the WT cell than the Ex6KO cell. As a result, the above-mentioned co-alignment of actin, vinculin and ERISM push-pull features was also less pronounced for the WT cell. In agreement with this, the forces exerted by single focal adhesions were smaller in the WT cell than in the Ex6KO cell (Fig. 5C,D). 
A

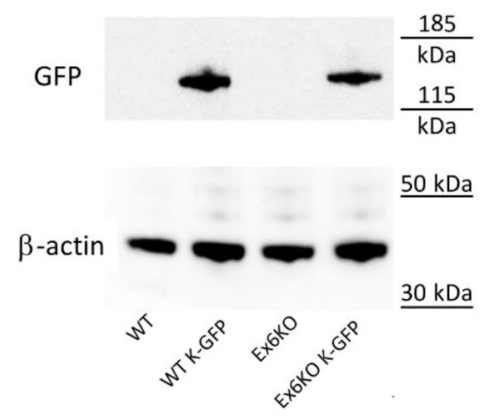

B

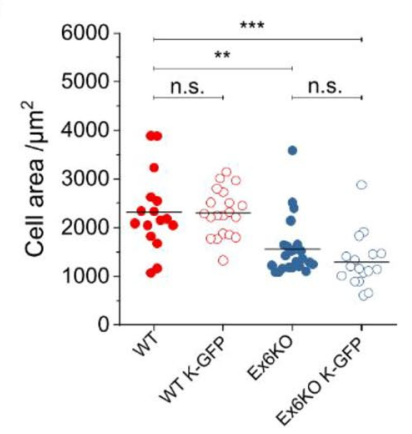

C

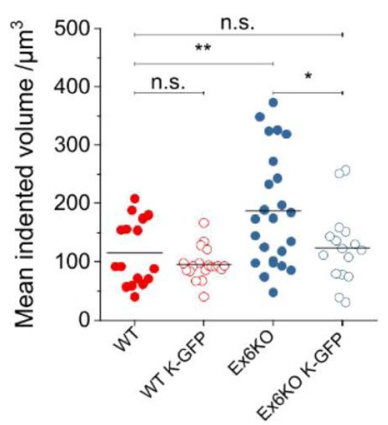

Figure 4. Phenotype recovery through KIAA0319 rescue. (A) Western blot confirming the presence of a fusion protein $(140 \mathrm{KDa}$ ) following transfection with a full length KIAA0319 construct fused to a GFP tag. Fulllength blots are presented in Supplementary Fig. S3. (B) Comparison of area covered by RPE1 WT, WT K-GFP, Ex6KO and Ex6KO K-GFP cells attached to ERISM micro-cavity. (WT: $n=16$, WT K-GFP: $n=20$, Ex6KO: $n=23$, Ex6KO KGFP: $n=17$ ). (C) Comparison of mean mechanical activity of RPE1 WT, WT K-GFP, Ex6KO and Ex6KO K-GFP cells during migration on ERISM micro-cavity. Only cells with free movement for $>4 \mathrm{~h}$ were included in the analysis. Plots in B and C show measured data points and the mean (line). (WT: $n=16$, WT K-GFP: $n=19$, Ex6KO: $n=24$, Ex6KO K-GFP: $n=16$ ) Groups were compared using the Student's t-test $\left({ }^{*} p \leq 0.05,{ }^{* *} p \leq 0.01,{ }^{* *} p \leq 0.001\right.$ ). Origin 2018 version $95 \mathrm{E}$ (https://www.originlab.com/2018) was used for data plotting and significance testing.

Besides the push-pull features associated with focal adhesions, the Fourier-filtered ERISM displacement maps also showed tightly confined pushing sites with a diameter of about $2 \mu \mathrm{m}$ (best visible as green-blue areas highlighted with black arrows in the Fourier-filtered ERISM map in inset i to Fig. 5A). These pushing sites were surrounded by circularly arranged dots of upward pulling (red areas highlighted with circles in inset i to Fig. 5A). Immunocytochemistry analysis showed that the pushing sites corresponded to actin-rich locations (white arrows in epi-fluorescence image in inset i to Fig. 5A; see also intensity profiles in Fig. S5), whereas pulling around the pushing sites colocalised with vinculin-rich positions (white circles in inset i to Fig. 5A; see also intensity profiles in Fig. S5). It has to be noted that the observed structures present poorly accumulating adhesive components and therefore more investigations will be required to fully understand their function.

The time-lapse measurement revealed that the actin dots exerted an oscillating vertical force that reached maximum values of up to $80 \mathrm{pN}$ (Fig. 5B). The horizontal contractile forces transmitted by focal adhesions were roughly 100-times larger than the vertical indentation forces exerted by the actin-rich protrusions (Fig. 5C,D). However, while the vertical pushing was highly dynamic, the horizontal forces originating from focal adhesions were relatively static and showed little oscillation in force. Focal adhesions at the leading edge of the cell were chiefly stationary once assembled (top right in Movie S4) and any lateral movement of focal adhesions was confined to the trailing edge of the cell (bottom left in Movie S4).

The WT and Ex6KO cell shown in Fig. 5, Movies S3 and S4 are examples illustrating the general differences between the two force transmission patterns (actin-rich protrusions and focal adhesions). In total, combined ERISM and immunochemistry measurements were carried out for 32 cells in three independent experiments (see Fig. S6, Movies S5, S6 for further examples). While both WT and Ex6KO cells formed protruding actin dots, we found that the number of cells with these structures were significantly lower for Ex6KO than for WT cells (fraction of cells forming actin dots \pm SEM: WT: $0.66 \pm 0.09, n=5,3$ and 4; Ex6KO: $0.11 \pm 0.11, n=5,6$ and $9, t$-test: $p=0.02$ ).

\section{Discussion}

We successfully developed a cellular knockout model via CRISPR-Cas9n to study the potential role of the KIAA0319 gene in cilia and cell migration on the basis of the proposed roles of this gene in the literature ${ }^{2}$. Overall, we found that loss of KIAA0319 in RPE1 cells results in elongation of the cilia and an increase of the force the cells apply on the substrate.

Although the same fraction of KIAA0319 knockout and WT cells developed cilia, these were significantly longer in the knockout (Fig. 2B). Mutations in the gene that encodes the cyclin-dependent kinase-like 5 (CDKL5) cause severe neurological symptoms and its knockdown in neurons produces cilia elongation ${ }^{28}$. Atypical cilia length has also been described for the knockdown of Nuclear Distribution Element 1 (NDE1), another gene associated with brain cortex development ${ }^{29}$. Cilia formation is a tightly regulated process, and cilia length has functional consequences on processes that include cell cycle re-entry and left-right patterning. Cilia biology is emerging as a contributing factor to a range of diseases, including neurodevelopmental disorders ${ }^{2}$. Other genes implicated in dyslexia have been reported to affect cilia length: knockouts for dyx1c1 present shorter cilia than the wild type in zebrafish ${ }^{30}$, and overexpression of Dcdc2 increases cilia length in rat neurons ${ }^{31}$. The only previous evidence in support of a role of KIAA0319 in cilia comes from transcriptomic studies ${ }^{9-11}$. Our work is therefore the first study to support a role for KIAA0319 in cilia biology in a biological model and paves the way for future studies aimed at dissecting the cellular function of this protein. 
A
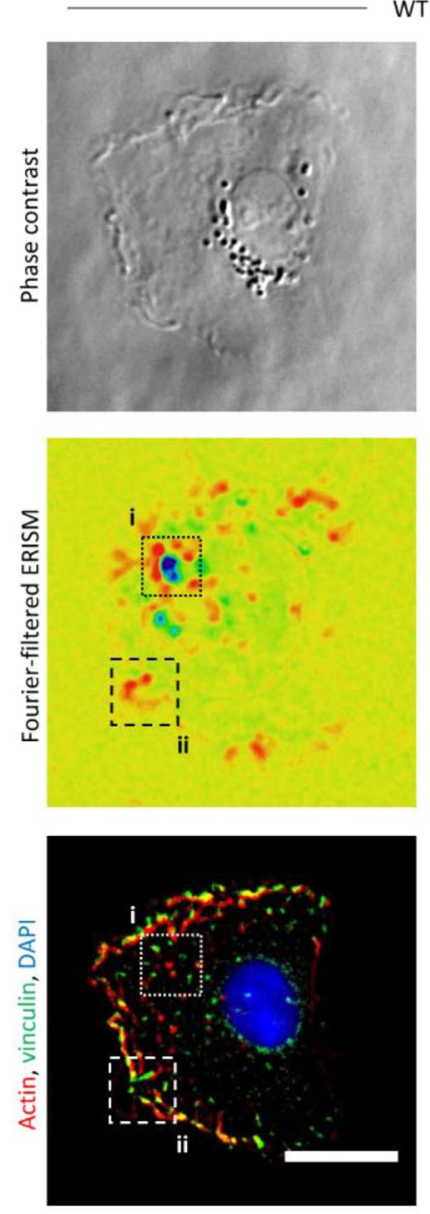

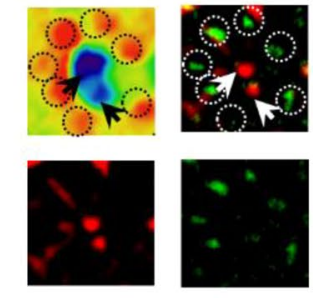

ii) Focal adhesions
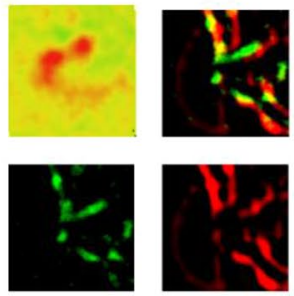

B

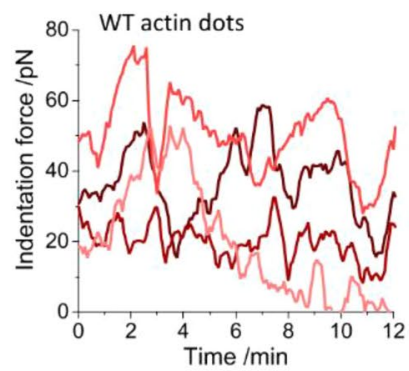

C

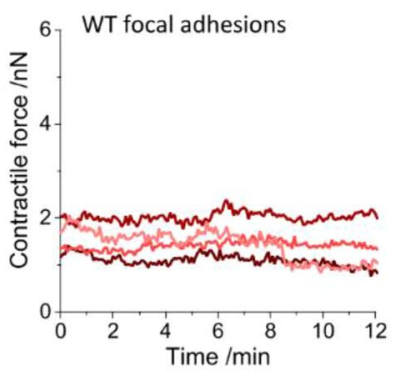

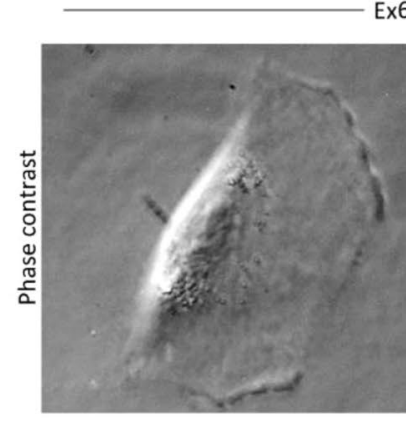

Ex6KO
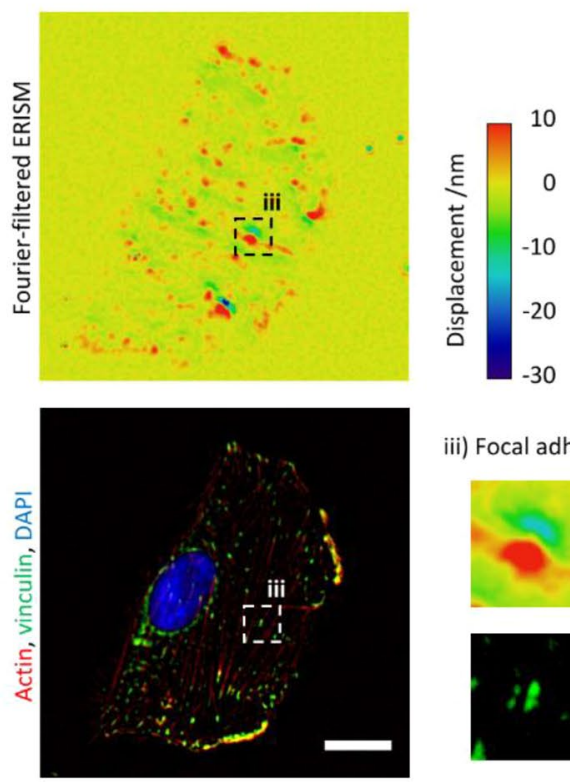

iii) Focal adhesions
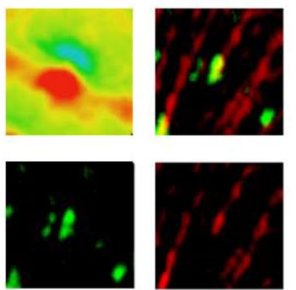

D

$E$
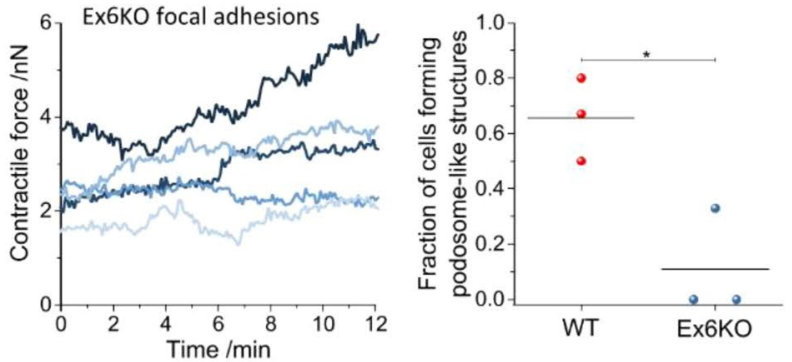

Figure 5. RPE1 KIAA0319 WT and Ex6KO cells use different modes of force exertion. (A) Phase contrast images (upper row), Fourier-filtered ERISM displacement maps (middle row) and epi-fluorescence images (lower row, red: actin, green: vinculin, blue: nuclear DNA) of a RPE1 WT cell (left column) and an Ex6KO cell (right column). The insets (i) in the Fourier-filtered ERISM map and the epi-fluorescence image of the WT cell show magnifications of protrusions linked to actin dots. Arrows indicate positions of actin-rich cell protrusions that are counterbalanced by pulling at vinculin-rich positions marked with circles. The insets (ii,iii) show magnifications of vinculin-rich cell-substrate contacts (focal adhesions) for the WT and Ex6KO cell, respectively. (B) Temporal evolution of the indentation force applied by different actin dots of the WT cell shown in (A). (C,D) Temporal evolution of the contraction force applied by different focal adhesions of the WT and Ex6KO cell shown in A, respectively. (E) Comparison of the fraction of WT and Ex6KO cells forming actin dot protrusions. Each data point represents the mean value of an independent experiment investigating $n=5$ (80\%), $3(67 \%)$ and $4(50 \%)$ WT and $n=5(0 \%), 6(0 \%)$ and $9(33 \%)$ Ex6KO cells, respectively. The lines depict the means. Groups were compared using the Student’s t-test $\left.{ }^{*} p \leq 0.05\right)$. Origin 2018 version 95E (https://www. originlab.com/2018) was used for data plotting and significance testing. All scale bars: $20 \mu \mathrm{m}$. 
Investigations on soft ERISM substrates showed that KIAA0319 knockout cells move significantly faster than wild type cells $(p=0.02$; Fig. 3C. However, when assessing collective cell migration with the commonly used scratch assay, we did not observe a significant effect of the KIAA0319 knockout (Fig. S1). Several factors might contribute to the different results obtained in these two experiments. First, individual cells might have different migration properties than a layer of collectively migrating cells. Second, the apparent stiffness of the ERISM sensor is in the range of soft tissue ( 1 to $20 \mathrm{kPa}$ ) and significantly different from the stiffness of the cell culture plastic plate in which the scratch assay was performed $(\sim 100,000 \mathrm{kPa})^{32}$. Substrate stiffness has a strong influence on cell migration in vitro ${ }^{33}$. Furthermore, while cells respond to an acute event, namely local damage, in the scratch assay, the ERISM assay observes the migration of undisturbed cells. Cell attachment proteins are another important factor in cell migration in vitro. Both the scratch assay and the ERISM measurements initially used serum containing media and we thus expect that proteins in the serum adhere to the substrate in both cases. However, in the case of the scratch assay, the medium was changed to serum free media after performing the scratch.

The ERISM analysis further revealed that the knockout cells exert significantly stronger forces on their substrate compared to the wild type (Fig. 3D). A rescue experiment in which a full length KIAA0319-GFP construct was overexpressed recovered the mechanical activity of the wild type phenotype (Fig. 4C), supporting an involvement of KIAA0319 in cellular forces. However, not all phenotypes observed in the Ex6KO cells were recovered, e.g. the reduction in cell area. Possible explanations for the partial recovery could be due to functions associated to specific KIAA0319 isoforms ${ }^{15}$, which were not restored in the rescue experiment. It is also possible that the GFP tag fused to KIAA0319 would interfere with the functionality of the protein for a well-known phenomenon ${ }^{34}$.

Fluorescent staining indicated the presence of actin-rich dots surrounded by vinculin-rich spots in both WT and KIAA0319 knockout cells (Fig. 5). By combining fluorescence staining with Fourier-filtered ERISM measurements, we found that the actin cores of these structures protruded vertically into the substrate, exerting oscillating forces of up to $80 \mathrm{pN}$, while surrounding rings of pulling sites were tightly colocalised with vinculin. While the in-depth characterization of these structures is beyond the scope of this work, we comment that the combination of this distinct force pattern and the protein arrangement of actin and vinculin have previously been associated with the presence of podosomes in macrophages ${ }^{22,24}$. Podosomes are cellular adhesion structures that are chiefly formed in monocyte-derived cells ${ }^{35}$ but that have also been reported in spreading and migrating epithelia cells ${ }^{36}$. While both WT and Ex6KO cells formed actin-rich protrusions, we found that the fraction of cells with these structures were significantly lower for KIAA0319 knockout than for WT cells (Fig. 5E).

KIAA0319 is a transmembrane protein that contains five PKD domains. These domains have been described in very few human proteins, the best characterised of which is Polycystin-1 (PC1). PC1 acts as a mechanosensor in the membrane of cilia $^{37}$, most probably by unfolding of the highly extensible PKD domains in response to stretching forces. It has been proposed that this unfolding maintains contact between neighbouring cells during cell movement ${ }^{38}$. PC1 interacts with the cytoskeleton ${ }^{39}$ and plays an important role in adaptative cilia shortening (for example under strong flow $)^{40}$. KIAA0319 might have a similar function to PC1, affecting cilia formation and possibly mechanosensing. The knockout of KIAA0319 not only results in formation of longer cilia, but also in an upregulation of mechanical forces and the reduced formation of actin-rich protrusions. The oscillating behaviour of these actin-rich protrusions might indicate that these structures play a role in mechanosensing as well-a function associated with podosomes ${ }^{35}$, which show a similar type of force exertion ${ }^{22,24}$. In epithelial cells, podosomes were reported to associate with hemidesmosomes ${ }^{36}$, adhesive structures specific to epithelial cells that regulate a wide range of biological processes including, among others, cell migration, exertion of traction force and mechanosensing ${ }^{36,41-44}$. The reduced formation of actin-rich protrusions in KIAA0319 knockout cells compared to the WT could support the hypothesis that KIAA0319 is involved in cellular mechanosensing. While it is tempting to speculate that PC1 and KIAA0319 may have similar functions due to the common appearance of PKD domains, further experiments are needed to substantiate the hypothesis that KIAA0319 is involved in mechanosensing.

The lack of specific antibodies able to detect endogenous levels of KIAA0319 in cell lines has greatly hampered the study of this protein. For this same reason we could not confirm the KO by western blotting. We addressed this limitation by validating the knockout with a combination of approaches: we confirmed loss-of-function deletions in the sixth exon of KIAA0319 that cause stop codons early in the transcript (exon 6 out of 21), so that the knockout cells cannot produce a functional protein. We detected a strong decrease in the expression of KIAA0319, consistent with nonsense-mediated decay of the transcript (Fig. 1D), a pathway which reduces errors in gene expression by eliminating mRNAs that contain premature stop codons, caused in our KIAA0319 knockout by loss-of-function deletions.

In summary, our work suggests that knockout of KIAA0319 affects processes controlled by the cytoskeleton such as cell migration, cilia length, cellular forces and the formation of actin-rich cell protrusions. Earlier studies showed that KIAA0319 overexpression inhibits axon growth and KIAA0319 knockout results in neurite outgrowth $^{18}$ - two further processes controlled by cytoskeleton filaments. As a transmembrane protein KIAA0319 might have an involvement in linking the cellular cytoskeleton to the extracellular matrix. Further work should therefore expand our studies on surfaces coated with specific extracellular matrix proteins. Due to its possible involvement in the regulation of cilia and cell forces, we speculate that the KIAA0319 gene might play a role during neurodevelopment. Such processes are being increasingly associated with neurodevelopmental disorders including schizophrenia, depression, bipolar disorder ${ }^{45}$ and autism ${ }^{46}$. 


\section{Materials and methods}

Cell culture. hTERT-RPE1 cells were generated by transfection with pGRN145, which expresses hTERT under the control of the MPSV promoter, and were kindly supplied by Dr. Andrea Bodnar, Geron Inc. Cell lines were cultured in complete media (DMEM F12 with 10\% of fetal bovine serum and $1 \%$ Penicillin/Streptomycin), or in serum-free media (DMEM F12 with $1 \%$ Penicillin/Streptomycin) at $37^{\circ} \mathrm{C}$ and $5 \% \mathrm{CO}_{2}$.

Plasmids. pSPgRNA was a gift from Charles Gersbach (Addgene plasmid \#47108) ${ }^{47}$. pSPCas9n-2A-GFP (pSpCas9n(BB)-2A-GFP (PX461)) was a gift from Feng Zhang (Addgene plasmid \#48140) ${ }^{48}$. KIAA0319-GFP was a gift from Antonio Velayos-Baeza ${ }^{15}$.

Cloning and transfection. KIAA0319 knockout cell lines were generated through a CRISPR-Cas9 double nicking strategy designed with the web-based tool developed by Hsu and collaborators (http://crispr.mit. edu $)^{49}$. This strategy uses Cas9 nickase (Cas9n), a modified Cas9 in which one of the nuclease domains has been mutated, lowering the rate of off-target effects compared to Cas9 ${ }^{48}$. RPE1 cells were transfected with pSpCas9n(BB)-2A-GFP (PX461) and paired gRNAs, using Lipofectamine3000 (ThermoFisher). gRNAs were generated by cloning annealed oligonucleotides containing the protospacer sequence into the chimeric gRNA sequence in pSPgRNA linearised with BbsI, downstream of a U6 promoter (Table S1). Sequences targeted were AGCCACCCCACAGACTACCA and TAAATTCCATTCATAGTTGT on KIAA0319 exon 6. pSpCas9n(BB)2A-GFP (PX461) contains a GFP expression cassette that acts as indicator of positive transfection. Twenty-four hours after transfection, 384 individual GFP positive cells (four 96 well plates) were isolated using Fluorescence Activated Cell Sorting (FACS) and plated onto 96 well plates coated with Poly-D-Lysine for clonal expansion.

Screening. Fifty cells were successfully expanded for further analysis. PCR was performed in all clones using primers int6-7R and int5-6F, that amplify a 1311 sequence DNA flanking the site targeted with the gRNAs (Table S1). Amplicons were digested with the restriction enzyme StyI. One of the used gRNAs targets this sequence, hence mutations caused by this gRNA are likely to eliminate this site. Amplicons from the 7 clones that showed loss of a StyI site upon digestion were cloned into Zero Blunt TOPO (ThermoFisher K280020) and sequenced using primers SP6 and T7. We identified one of these lines as a homozygous knockout as it contains two types of deletions causing frameshifts and premature stop codons.

Immunofluorescence. Cells on the ERISM micro-cavity were fixed with $4 \%$ paraformaldehyde (PFA) in $\mathrm{PBS}$ at room temperature for $20 \mathrm{~min}$. Immediately after fixation, cells were permeabilised with $0.1 \%$ Triton X-100 for $3 \mathrm{~min}$ and blocked for $30 \mathrm{~min}$ with 1\% BSA in PBS. Cells were then stained for vinculin using anti-vinculin antibody (Merck Millipore, cat. no. 90227, 1:250 in BSA solution, $1 \mathrm{~h}$ at room temperature) followed by a secondary anti-mouse antibody (FITC-conjugated, Sigma-Aldrich, F0257 or Alexa Fluor 647-conjugated, Invitrogen, A32728), and for actin using TRITC-conjugated phalloidin (MerckMillipore, cat. no. 90228, 1:500 in BSA solution, $1 \mathrm{~h}$ at room temperature). The nuclei of the cells were stained with DAPI (MerckMillipore, 1:1000 in BSA), at room temperature for $3 \mathrm{~min}$.

RPE1 cells for cilia analysis were cultured on uncoated coverslips for $48 \mathrm{~h}$ with serum-free media, fixed with $4 \%$ PFA for 10 min, permeabilised with $0.1 \%$ Triton X-100, blocked with $10 \%$ goat serum in PBS, and stained with the ciliary marker ARL13B Antibody Rabbit polyclonal (17711-1-AP Proteintech) and Mouse Monoclonal anti-gamma-tubulin (Abcam 11316) followed by secondary antibodies anti-rabbit Alexa Fluor 488 and antimouse Alexa Fluor 594 (Invitrogen). Under serum starvation, cells stay in $\mathrm{G}_{0}$ and form cilia. We measured the length of the cilia manually using ImageJ. To ensure that cilia were positioned flat against the surface of the cell, only cilia that were completely in focus were considered.

Gene expression quantification. qRT-PCRs were performed using Luna OneStep reagent (NEB) on biological triplicates. KIAA0319 expression was assessed with primers ex11F and ex12R (Table S1). Analysis was performed by the $\Delta \Delta \mathrm{Ct}$ method using Beta-actin as endogenous control. Results were normalised against expression in WT cells. Error bars are calculated using the standard deviation of the triplicates $\left(2^{\Delta \Delta \mathrm{Ct}-\mathrm{s} . \mathrm{d}}-2^{\Delta \Delta \mathrm{Ct}+-\mathrm{s} . \mathrm{d}}\right)$.

Western blot. Protein lysates were obtained from all cell lines using RIPA buffer and separated in a NuPAGE Bis-Tris 4-12\% gradient gel (ThermoFisher). Proteins were transferred to a nitrocellulose membrane, blocked in WesternBreeze blocker (ThermoFisher) and incubated with primary antibodies anti-GFP (Chromotek \#029762) and anti-beta actin (Sigma). Secondary antibodies were donkey anti-rat and anti-mouse HRP conjugated. Membranes were developed using SuperSignal WestFemto substrate (ThermoFisher).

Scratch assay. The scratch assay is a simple way to measure cell migration in vitro and consists on creating a "scratch" on a confluent layer of cells and quantifying the movement of the cells over time to close this gap ${ }^{50}$. Since this test is performed in serum free culture conditions, which prevent the cells from dividing, it only takes into account cell movement and not proliferation. Wild type and Ex6KO cell lines were plated on a 6-well plastic plate (Nunclon Delta Surface, ThermoFisher Scientific). When confluent, the layer of cells was scratched with a pipette tip creating a straight gap. Cells were then washed with PBS to remove media and floating cells, and serum free media was added. We took images covering the whole gap at the time of the scratch (time 0 ) and after $24 \mathrm{~h}$. We measured the width of the scratch using TScratch ${ }^{51}$, and calculated the mean width for each cell line after $24 \mathrm{~h}$. 
ERISM measurements. ERISM substrates were fabricated as described previously ${ }^{22}$ and four silicon chambers (surface area: $0.75 \times 0.75 \mathrm{~cm}^{2}$; Ibidi) were applied. RPE1 cells were seeded on the ERISM substrate at 1,000 cells per well and kept at $37^{\circ} \mathrm{C}, 5 \% \mathrm{CO}_{2}$ culture conditions in DMEM-12 supplemented with $10 \%$ FBS and $1 \%$ Penicillin/Streptomycin. WT and Ex6KO cells as well as WT, WT_K-GFP, Ex6KO and Ex6KO_K-GFP cells were investigated in different wells on the same ERISM chip. Prior to ERISM measurements, cells were cultured for $24 \mathrm{~h}$ to allow adhesion to complete. ERISM force measurements were performed and converted into displacement maps as described before ${ }^{22}$. To investigate forces during cell migration, ERISM maps were recorded continuously for $17 \mathrm{~h}$ at intervals of $5 \mathrm{~min}$, recording from seven different positions within each of the respective wells with $\mathrm{a} \times 20$ objective. To analyse the force exertion patterns, ERISM measurements were performed at higher frame rate (every $5 \mathrm{~s}$ or $2 \mathrm{~min}$ ) and magnification ( $\times 40$ objective). To generate the Fourier-filtered ERISM maps, a FFT bandpass filter was applied to the raw displacement maps using the ImageJ software. For cell force analysis, the volume by which migrating cells indent into the ERISM substrate was calculated using ImageJ. All pixels in the ERISM displacement maps with indentation of less than $20 \mathrm{~nm}$ were set to NaN's (not a number) and the "indented volume" under each individual cell was calculated as the product of area and mean indentation. Only cells that moved freely for $\geq 2 \mathrm{~h}$ (i.e. that were not in physical contact with other cells) were included in the analysis.

The "indentation force" of a single actin-rich protrusion was calculated by converting spatial Fourier-filtered ERISM displacement maps with a cut-off frequency of $0.6 \mu \mathrm{m}^{-1}$ into stress maps using FEM as described in Ref. ${ }^{22}$. These protrusions were identified in stress maps as isolated, localised indentation surrounded by a ring of pulling. Indentation force was calculated as the product of indentation area and mean applied stress at a threshold of $4 \mathrm{~Pa}$. Only structures that colocalise with actin-dots in the respective immunostaining image were analysed.

To calculate the "contraction force" of single focal adhesions, the twist in spatial Fourier-filtered ERISM displacement maps with a cut-off frequency of $0.6 \mu \mathrm{m}^{-1}$ were analysed and converted into the corresponding horizontally exerted contractile forces as described in Ref. ${ }^{22}$. In short, twisting results from the torque applied by focal adhesions when transmitting contractile cell forces to the ERISM substrate. The twisting response of ERISM substrates was calibrated by applying horizontal forces using AFM. The amount of twisting was found to be directly proportional to the applied force $\left(6.6 \mathrm{~nm}\right.$ of twist per $1 \mathrm{nN}$ of applied force; $R^{2}>0.99 ; n=5$ force measurements). Only twists in ERISM displacement maps that form around vinculin-rich areas in the respective immunostaining image were analysed.

The speed of the cells on the ERISM sensor was measured using the plugin Manual Tracking on ImageJ ${ }^{21}$. The "straightness" of cell migration was calculated as the ratio of the effective displacement of the cell relative to the position at the start of the measurement and the track length. Cell areas were measured from single phase contrast images by drawing the outline of the cells in ImageJ.

Generation of cell lines expressing KIAA0319-GFP. RPE1 wild type and Ex6KO were transfected with linearised KIAA0319-GFP plasmid using Lipofectamine 3000 according to the manufacturer's specifications. KIAA0319-GFP contains a neomycin resistance cassette that was used to select cells that had undergone stable transfection, integrating the construct in their genome. Stably transfected cells were selected with G418 (Roche) at a concentration of $400 \mu \mathrm{g} \mathrm{ml}^{-1}$ for 2 weeks. Cells tend to lose the expression of the transgene with time $\mathrm{e}^{52}$, and after a few passages of this cell line, GFP expression was detected in only a small percentage of the cells. To enrich cells expressing the construct, we selected GFP positive cells via FACS. After FACS selection, cells were kept in culture for $24 \mathrm{~h}$ to allow them to recover, and then plated onto the ERISM microcavity for measurement.

\section{Data availability}

The research data underpinning this publication can be accessed at https://doi.org/10.17630/3eb826ac-eea7478e-b6dfee4dd50a96fd.

Received: 5 April 2021; Accepted: 17 December 2021

Published online: 14 January 2022

\section{References}

1. Peterson, R. L. \& Pennington, B. F. Developmental dyslexia. Lancet 379, 1997-2007. https://doi.org/10.1016/S0140-6736(12) 60198-6 (2012).

2. Paracchini, S., Diaz, R. \& Stein, J. Advances in dyslexia genetics-New insights into the role of brain asymmetries. Adv. Genet. https://doi.org/10.1016/bs.adgen.2016.08.003 (2016).

3. Paracchini, S., Scerri, T. \& Monaco, A. P. The genetic lexicon of dyslexia. Annu. Rev. Genomics Hum. Genet. 8, 57-79. https://doi. org/10.1146/annurev.genom.8.080706.092312 (2007).

4. Galaburda, A. M., Sherman, G. F., Rosen, G. D., Aboitz, F. \& Geschwind, N. Developmental dyslexia: Four consecutive patients with cortical anomaly. Ann. Neurol. 18, 222-223. https://doi.org/10.1002/ana.410180210 (1985).

5. Guidi, L. G. et al. The neuronal migration hypothesis of dyslexia: A critical evaluation thirty years on. Eur. J. Neurosci. 48, 32123233. https://doi.org/10.1111/ejn.14149 (2018).

6. Rosen, G. D. et al. Disruption of neuronal migration by RNAi of Dyxlcl results in neocortical and hippocampal malformations. Cereb. Cortex 17, 2562-2572. https://doi.org/10.1093/cercor/bhl162 (2007).

7. Galaburda, A. M. The role of rodent models in dyslexia research: Understanding the brain, sex differences, lateralization, and behavior. In Reading and Dyslexia: From Basic Functions to Higher Order Cognition (eds Lachmann, T. \& Weis, T.) 77-96 (Springer, 2018).

8. Centanni, T. M. Neural and genetic mechanisms of dyslexia. In Translational Neuroscience of Speech and Language Disorders (ed. Argyropoulos, G. P. D.) 47-68 (Springer, 2020).

9. Geremek, M. et al. Ciliary genes are down-regulated in bronchial tissue of primary ciliary dyskinesia patients. PLoS ONE. https:// doi.org/10.1371/journal.pone.0088216 (2014). 
10. Hoh, R. A., Stowe, T. R., Turk, E. \& Stearns, T. Transcriptional program of ciliated epithelial cells reveals new cilium and centrosome components and links to human disease. PLoS ONE 7, e52166. https://doi.org/10.1371/journal.pone.0052166 (2012).

11. Ivliev, A. E., 't Hoen, P. A. C., van Roon-Mom, W. M. C., Peters, D. J. M. \& Sergeeva, M. G. Exploring the transcriptome of ciliated cells using in silico dissection of human tissues. PLoS ONE 7, e35618. https://doi.org/10.1371/journal.pone.0035618 (2012).

12. Brandler, W. M. \& Paracchini, S. The genetic relationship between handedness and neurodevelopmental disorders. Trends Mol. Med. 20, 1-8. https://doi.org/10.1016/j.molmed.2013.10.008 (2013).

13. Pruski, M. \& Lang, B. Primary cilia-An underexplored topic in major mental illness. Front. Psychiatry. https://doi.org/10.3389/ FPSYT.2019.00104 (2019).

14. Velayos-Baeza, A., Toma, C., da Roza, S., Paracchini, S. \& Monaco, A. P. Alternative splicing in the dyslexia-associated gene KIAA0319. Mamm. Genome 18, 627-634. https://doi.org/10.1007/s00335-007-9051-3 (2007).

15. Velayos-Baeza, A., Toma, C., Paracchini, S. \& Monaco, A. P. The dyslexia-associated gene KIAA0319 encodes highly N- and O-glycosylated plasma membrane and secreted isoforms. Hum. Mol. Genet. 17, 859-871. https://doi.org/10.1093/hmg/ddm358 (2008).

16. Ibraghimov-Beskrovnaya, O. et al. Strong homophilic interactions of the Ig-like domains of polycystin-1, the protein product of an autosomal dominant polycystic kidney disease gene, PKD1. Hum. Mol. Genet. 9, 1641-1649 (2000).

17. Velayos-Baeza, A., Levecque, C., Kobayashi, K., Holloway, Z. G. \& Monaco, A. P. The dyslexia-associated KIAA0319 protein undergoes proteolytic processing with \{gamma\}-secretase-independent intramembrane cleavage. J. Biol. Chem. 285, 40148-40162. https://doi.org/10.1074/jbc.M110.145961 (2010).

18. Franquinho, F. et al. The dyslexia-susceptibility protein KIAA0319 inhibits axon growth through Smad2 signaling. Cereb. Cortex 27, 1732-1747. https://doi.org/10.1093/cercor/bhx023 (2017).

19. Gostic, M. et al. The dyslexia susceptibility KIAA0319 gene shows a specific expression pattern during zebrafish development supporting a role beyond neuronal migration. J. Comp. Neurol. 527, 1-10. https://doi.org/10.1002/cne.24696 (2019).

20. De, Wu. G., Li, Z. H., Li, X., Zheng, T. \& Zhang, D. K. microRNA-592 blockade inhibits oxidative stress injury in Alzheimer's disease astrocytes via the KIAA0319-mediated Keap1/Nrf2/ARE signaling pathway. Exp. Neurol. 324, 113128. https://doi.org/10. 1016/j.expneurol.2019.113128 (2020).

21. Schneider, C. A., Rasband, W. S. \& Eliceiri, K. W. NIH Image to ImageJ: 25 years of image analysis. Nat. Methods 9, 671-675 (2012).

22. Kronenberg, N. M. et al. Long-term imaging of cellular forces with high precision by elastic resonator interference stress microscopy. Nat. Cell Biol. 19, 864-872. https://doi.org/10.1038/ncb3561 (2017).

23. Liehm, P., Kronenberg, N. M. \& Gather, M. C. Analysis of the precision, robustness, and speed of elastic resonator interference stress microscopy. Biophys. J. 114, 2180-2193. https://doi.org/10.1016/j.bpj.2018.03.034 (2018).

24. Labernadie, A. et al. Protrusion force microscopy reveals oscillatory force generation and mechanosensing activity of human macrophage podosomes. Nat. Commun. 5, 5343. https://doi.org/10.1038/ncomms6343 (2014).

25. Baker, K. E. \& Parker, R. Nonsense-mediated mRNA decay: Terminating erroneous gene expression. Curr. Opin. Cell Biol. 16, 293-299. https://doi.org/10.1016/J.CEB.2004.03.003 (2004).

26. Levecque, C., Velayos-baeza, A. \& Holloway, Z. G. The dyslexia-associated protein KIAA0319 interacts with adaptor protein 2 and follows the classical clathrin-mediated endocytosis pathway. Am. J. Physiol. Cell Physiol. 2, 160-168. https://doi.org/10.1152/ajpce 11.00630 .2008 (2009).

27. Legant, W. R. et al. Multidimensional traction force microscopy reveals out-of-plane rotational moments about focal adhesions. Proc. Natl. Acad. Sci. 110, 1-6. https://doi.org/10.1073/pnas.1207997110 (2013).

28. Di Nardo, A. et al. Phenotypic characterization of Cdkl5-knockdown neurons establishes elongated cilia as a functional assay for CDKL5 deficiency disorder. Neurosci. Res. https://doi.org/10.1016/j.neures.2021.10.001 (2021).

29. Kim, S. et al. Nde1-mediated inhibition of ciliogenesis affects cell cycle re-entry. Nat. Cell Biol. 13, 351-362. https://doi.org/10. 1038/ncb2183 (2011).

30. Chandrasekar, G., Vesterlund, L., Hultenby, K., Tapia-Páez, I. \& Kere, J. The zebrafish orthologue of the dyslexia candidate gene DYX1C1 is essential for cilia growth and function. PLoS ONE 8, e63123. https://doi.org/10.1371/journal.pone.0063123 (2013).

31. Massinen, S. et al. Increased expression of the dyslexia candidate gene DCDC2 affects length and signaling of primary cilia in neurons. PLoS ONE 6, e20580. https://doi.org/10.1371/journal.pone.0020580 (2011).

32. Skardal, A., Mack, D., Atala, A. \& Soker, S. Substrate elasticity controls cell proliferation, surface marker expression and motile phenotype in amniotic fluid-derived stem cells. J. Mech. Behav. Biomed. Mater. 17, 307-316. https://doi.org/10.1016/j.jmbbm.2012. 10.001 (2013).

33. Bangasser, B. L. et al. Shifting the optimal stiffness for cell migration. Nat. Commun. 8, 15313. https://doi.org/10.1038/ncomm s15313 (2017).

34. Snapp, E. L. Fluorescent proteins: A cell biologist's user guide. Trends Cell Biol. 19, 649-655. https://doi.org/10.1016/j.tcb.2009.08. 002.Fluorescent (2009).

35. Linder, S. \& Wiesner, C. Feel the force: Podosomes in mechanosensing. Exp. Cell Res. 343, 67-72. https://doi.org/10.1016/J.YEXCR. 2015.11.026 (2016).

36. Spinardi, L. et al. A dynamic podosome-like structure of epithelial cells. Exp. Cell Res. 295, 360-374. https://doi.org/10.1016/j. yexcr.2004.01.007 (2004).

37. Dalagiorgou, G., Basdra, E. K. \& Papavassiliou, A. G. Polycystin-1: Function as a mechanosensor. Int. J. Biochem. Cell Biol. 42, 1610-1613. https://doi.org/10.1016/J.BIOCEL.2010.06.017 (2010).

38. Qian, F., Wei, W., Germino, G. \& Oberhauser, A. The nanomechanics of polycystin-1 extracellular region. J. Biol. Chem. 280, 40723-40730. https://doi.org/10.1074/jbc.M509650200 (2005).

39. Boca, M. et al. Polycystin-1 induces cell migration by regulating phosphatidylinositol 3-kinase-dependent cytoskeletal rearrangements and GSK3-dependent cell-cell mechanical adhesion. Mol. Biol. Cell 18, 4050-4061. https://doi.org/10.1091/mbc.E07-02-0142 (2007).

40. Besschetnova, T. Y. et al. Identification of signaling pathways regulating primary cilium length and flow-mediated adaptation. Curr. Biol. 20, 182-187. https://doi.org/10.1016/J.CUB.2009.11.072 (2010).

41. Hiroyasu, S., Colburn, Z. T. \& Jones, J. C. R. A hemidesmosomal protein regulates actin dynamics and traction forces in motile keratinocytes. FASEB J. 30, 2298-2310. https://doi.org/10.1096/f.201500160R (2016).

42. Zhang, H. et al. A tension-induced mechanotransduction pathway promotes epithelial morphogenesis. Nature 471, 99-103. https:// doi.org/10.1038/nature09765 (2011).

43. Grashoff, C. et al. Measuring mechanical tension across vinculin reveals regulation of focal adhesion dynamics. Nature 466, 263-266. https://doi.org/10.1038/nature09198 (2010).

44. Walko, G., Castañón, M. J. \& Wiche, G. Molecular architecture and function of the hemidesmosome. Cell Tissue Res. 360, 363-378. https://doi.org/10.1007/s00441-014-2061-z (2015).

45. Marchisella, F., Coffey, E. T. \& Hollos, P. Microtubule and microtubule associated protein anomalies in psychiatric disease. Cytoskeleton 73, 596-611. https://doi.org/10.1002/cm.21300 (2016).

46. Lin, Y.-C., Frei, J. A., Kilander, M. B. C., Shen, W. \& Blatt, G. J. A Subset of autism-associated genes regulate the structural stability of neurons. Front. Cell Neurosci. 10, 263. https://doi.org/10.3389/fncel.2016.00263 (2016).

47. Perez-Pinera, P. et al. RNA-guided gene activation by CRISPR-Cas9-Based transcription factors. Nat. Methods 10, 973-976. https://doi.org/10.1038/NMETH.2600 (2013). 
48. Ran, F. A. et al. Genome engineering using the CRISPR-Cas9 system. Nat Protoc. 8, 2281-2308. https://doi.org/10.1038/nprot. 2013.143 (2013).

49. Hsu, P. D. et al. DNA targeting specificity of RNA-guided Cas9 nucleases. Nat. Biotechnol. 31, 827-832. https://doi.org/10.1038/ nbt.2647 (2013).

50. Liang, C. C., Park, A. Y. \& Guan, J. L. In vitro scratch assay: A convenient and inexpensive method for analysis of cell migration in vitro. Nat. Protoc. 2, 329-333. https://doi.org/10.1038/nprot.2007.30 (2007).

51. Geback, T., Schulz, M. M. P., Koumoutsakos, P. \& Detmar, M. TScratch: A novel and simple software tool for automated analysis of monolayer wound healing assays. Biotechniques 46, 265-274. https://doi.org/10.2144/000113083 (2009).

52. Mutskov, V. \& Felsenfeld, G. Silencing of transgene transcription precedes methylation of promoter DNA and histone H3 lysine 9. EMBO J. 23, 138-149. https://doi.org/10.1038/sj.emboj.7600013 (2004).

\section{Acknowledgements}

We thank Dr. Samantha Pitt and Dr. Swati Arya for their suggestions to improve the manuscript.

\section{Author contributions}

R.D. and N.M.K. conducted the experiments and analysed the data with support from A.M. and P.L. A.C.R. assisted with the generation of the cell lines. R.D. and N.M.K. wrote the manuscript with input from all authors. M.C.G. and S.P. co-supervised the work.

\section{Funding}

This work was supported by Action Medical Research/The Chief Scientist (CSO) Office, Scotland [GN 2614], Royal Society [RG160373], Carnegie Trust [50341], Wellcome Trust ISSF [105621/Z/14/Z], and RS Macdonald Charitable Trust Grants to SP and Engineering and Physical Sciences Research Council [EP/P030017/1], Biotechnology and Biological Sciences Research Council [BB/P027148/1], and the European Research Council Starting Grant ABLASE [640012] Grants to MCG.

\section{Competing interests}

The authors declare no competing interests.

\section{Additional information}

Supplementary Information The online version contains supplementary material available at https://doi.org/ 10.1038/s41598-021-04539-3.

Correspondence and requests for materials should be addressed to M.C.G. or S.P.

Reprints and permissions information is available at www.nature.com/reprints.

Publisher's note Springer Nature remains neutral with regard to jurisdictional claims in published maps and institutional affiliations.

(c) (i) Open Access This article is licensed under a Creative Commons Attribution 4.0 International cc) License, which permits use, sharing, adaptation, distribution and reproduction in any medium or format, as long as you give appropriate credit to the original author(s) and the source, provide a link to the Creative Commons licence, and indicate if changes were made. The images or other third party material in this article are included in the article's Creative Commons licence, unless indicated otherwise in a credit line to the material. If material is not included in the article's Creative Commons licence and your intended use is not permitted by statutory regulation or exceeds the permitted use, you will need to obtain permission directly from the copyright holder. To view a copy of this licence, visit http://creativecommons.org/licenses/by/4.0/.

(c) The Author(s) 2022 